\title{
Neurology of endemic skeletal fluorosis
}

\author{
D. Raja Reddy \\ Department of Neurosurgery, Room No. 39, Apollo Hospital, Jubilee Hills, Hyderabad - 500 033, India
}

\begin{abstract}
Endemic skeletal fluorosis is widely prevalent in India and is a major public health problem. The first ever report of endemic skeletal fluorosis and neurological manifestation was from Prakasam district in Andhra Pradesh in the year 1937. Epidemiological and experimental studies in the endemic areas suggest the role of temperate climate, hard physical labor, nutritional status, presence of abnormal concentrations of trace elements like strontium, uranium, silica in water supplies, high fluoride levels in foods and presence of kidney disease in the development of skeletal fluorosis. Neurological complications of endemic skeletal fluorosis, namely radiculopathy, myelopathy or both are mechanical in nature and till date the evidence for direct neurotoxicity of fluoride is lacking. Prevention of the disease should be the aim, knowing the pathogenesis of fluorosis. Surgery has a limited role in alleviating the neurological disability and should be tailored to the individual based on the imaging findings.
\end{abstract}

Address for correspondence:

D. Raja Reddy,

Department of Neurosurgery,

Room No. 39, Apollo Hospital,

J ubilee Hills, Hyderabad - 500 033,

India.

E-mail: palvashareddy@ gmail

DOI: $10.4103 / 0028-3886.48793$
Key words: Endemic skeletal fluorosis, fluoride, myelopathy, radiculopathy, spinal decompressive surgery, strontium

\section{Introduction}

Endemic skeletal fluorosis is widely prevalent in India and in many countries around the world but it is a major public health problem in some 25 countries in Asia and Africa [Figure 1, Figure 2]. ${ }^{[1]}$ Sixty million Indians are living in about 200 districts of 20 states in endemic areas of fluorosis and are at risk of developing fluorosis [Table 1]..$^{[2]}$ In India there are about six million people disabled because of fluorosis and a tenth of those might develop neurological sequelae. Skeletal fluorosis is caused by prolonged intake of excessive amounts of fluoride through water, food or both. The optimum upper safe limit is not more than 6 milligrams per day. ${ }^{[3]}$ Epidemiological and experimental studies on fluorosis have greatly helped in understanding the disease and provided a rational approach of management of the menace of fluoride toxicity.

\section{Skeletal Fluorosis - Pathogenesis}

The first ever report of endemic skeletal fluorosis was from Podili, Darsi and Kanigiri areas of Prakasam district in Andhra Pradesh, a province in south India, in the year 1937. ${ }^{[4]}$ This report also had the description of neurological manifestations of fluorosis, a manifestation of late stage of fluoride toxicity. Surprisingly, the fluoride levels of drinking water in these areas were not very high and were in the range of 1-3 PPM and rarely exceeded 6 PPM. ${ }^{[5]}$ Development of skeletal fluorosis has not been reported with such levels of fluoride in drinking water from other parts of the world. The proposed possible mechanisms for such severe manifestations by the authors included: (a) high atmospheric temperatures $\left(115-116^{\circ} \mathrm{F}\right)$ during summer months; (b) hard physical labor activity (both factors are

\begin{tabular}{lc}
\hline \multicolumn{2}{l}{ Table 1: Number of distric ts known to be endemic for fluorosis } \\
in various states of India \\
\hline Assam & 02 \\
Andhra Pradesh & 17 \\
Bihar & 08 \\
Delhi & 04 \\
Gujarat & All except 1 \\
Haryana & 12 \\
Jammu \& Kashmir & 01 \\
Karnataka & 13 \\
Kerala & 03 \\
Maharashtra & 10 \\
Orissa & 03 \\
Punjab & 13 \\
Rajasthan & All \\
Tamil Nadu & 08 \\
Uttar Pradesh & 07 \\
West Bengal & 04 \\
\hline
\end{tabular}




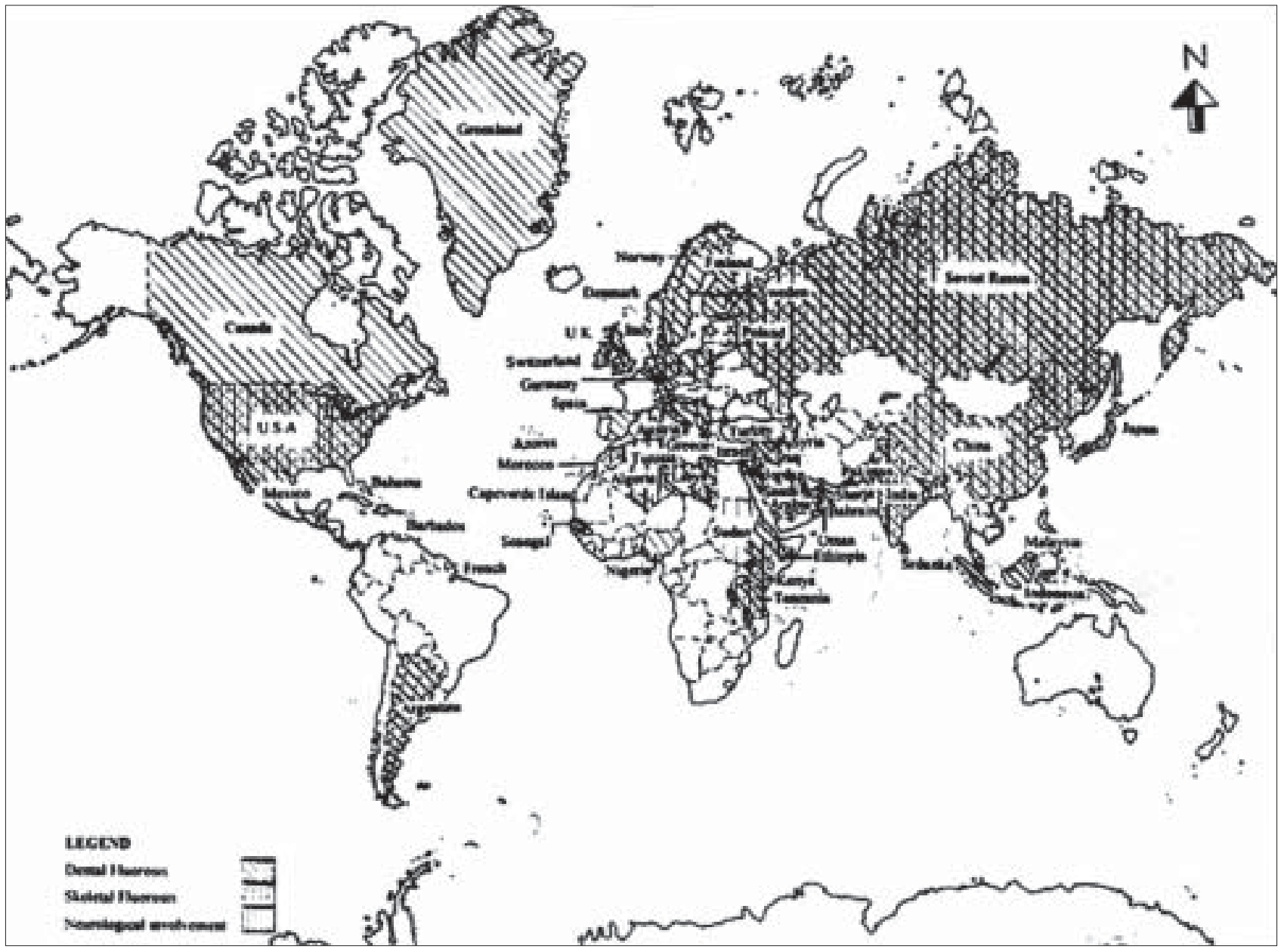

Figure 1: World map showing dental, skeletal and neurological fluorosis in different countries

likely to lead to high water intake); (c) poor nutrition, deficient in calories and also vitamin $\mathrm{C}$. What was not realized then was that the ten patients reported by Shortt and colleagues had impaired renal functions. ${ }^{[4]}$ It is now established that diseased kidneys cannot handle fluoride excretion leading to fluoride toxicity and development of skeletal fluorosis even while consuming low levels of fluoride in drinking water supplies. ${ }^{[6]}$ Recent studies also suggest that the presence of certain trace elements like strontium, silica, uranium, calcium, magnesium etc., in high concentration in water and food could influence fluoride toxicity, some beneficial and others detrimental. ${ }^{[7,8]}$ Ninety-four drinking water samples from endemic areas in Prakasam district were investigated for the content of fluoride and strontium. Fluoride levels ranged between 0.1-9.5 PPM with mean fluoride content of 2.1 PPM whereas strontium levels varied between 4.9-9931.7 PPB with a mean of 1591.2 PPB. Strontium is a common element which is naturally found in bones, it inhibits bone resorption while simultaneously stimulating bone growth. It is possible that this high water content of strontium may be playing role in fluoride toxicity in the endemic areas in Prakasam district. The above observations suggest that the factors that may predispose to the development of endemic skeletal fluorosis include: (a) high fluoride intake through water and food; (b) continued exposure to fluoride; (c) strenuous manual physical activity; (d) poor nutrition; (e) impaired renal function and (f) abnormal concentrations of certain trace elements.

\section{Clinical Features}

Fluoride toxicity presents with an extraordinary degree of uniformity in its clinical manifestations. Fluorosis in humans is predominantly dental and skeletal. These two forms are separated by a prolonged relatively symptom-free interval of 10-30 years during which time the skeleton does not stop accumulating fluoride though non-skeletal symptoms such as gastrointestinal may be present. In the advanced stages, skeletal fluorosis causes crippling deformities and neurological complications. The neurological manifestations in skeletal fluorosis, radiculo-myelopathy, arise primarily from mechanical compression of the spinal cord and 


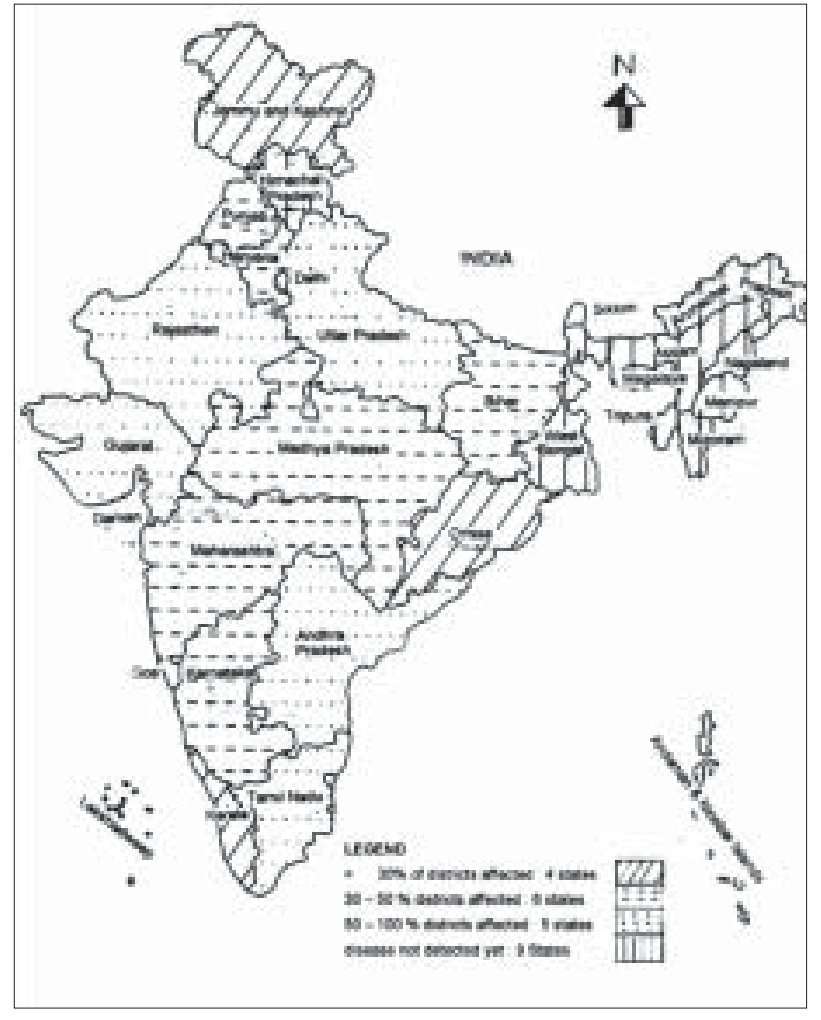

Figure 2: Intensity of fluorosis in different states of India

nerve roots from osteophytosis, sclerosed vertebral column and ossified ligaments. In the later stages as the disease progresses there may be vascular compromise and ischemic injury of spinal cord and spinal roots. In skeletal fluorosis cervical cord is affected earlier than the dorsal cord. Though lumbar spine is the first to exhibit skeletal changes, somehow compression of cauda equina is rare. It may be related to the course of the lumbo-sacral roots. The neurological deficits may sometimes be precipitated by a minor trauma. Neurological deficits are often associated with restricted spinal movement. In skeletal fluorosis higher cerebral function defects or cranial nerve palsies are extremely rare.

Neurophysiological experiments have revealed that sodium fluoride has anticholinesterase and anticurarelike effects on muscle and nerve, although it has no effect on normal muscle membrane potentials even in the endplate region. ${ }^{[9]}$ There is yet no evidence of direct neurotoxicity of fluoride. Detailed histological and histochemical studies of muscle biopsies in patients suffering from endemic skeletal fluorosis showed no evidence of direct toxic effect and the muscle changes were neurogenic, secondary to compressive radicluopathy. ${ }^{[10]}$ Sural nerve biopsies in patients with endemic skeletal fluorosis showed reduced myelinated fiber densities indicating a dropout probably due to axonal degeneration or demyelination or both. ${ }^{[1]}$
The pathological findings are further supported by the electrodiagnostic studies which included nerve conduction studies, late responses ${ }^{[12]}$ and electromyography. ${ }^{[13]}$

There are hardly any autopsy studies in skeletal fluorosis. Franke reported anterior horn cell damage in spinal cord in a patient with skeletal fluorosis and attributed the changes to the direct toxic effect of fluoride. There was no compressive pathology of cord or nerves in this patient. ${ }^{[14]}$ Study of spinal cord in fluorotic dogs from endemic regions without myelographic evidence of compression showed no histological changes to suggest anterior horn cell

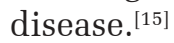

\section{Diagnosis}

Urinary and bone fluoride

Urinary fluoride levels are the best indicators of fluoride intake. Since fluoride excretion is not constant throughout the day, 24-h samples of urine are more reliable than random or morning samples. ${ }^{[16,17]}$ In normal individuals urinary fluorides fluctuates widely between 0.1 to 2.0 PPM (average 0.4 PPM) when the fluoride content of drinking water is $0.3 \mathrm{PPM}$. There is a linear relation between urinary fluoride levels and fluoride intake. ${ }^{[17]}$ Normal blood fluoride levels in non-endemic regions varied between 0.002 and $0.008 \mathrm{mg} / 100 \mathrm{ml}$ and in endemic regions the levels range between 0.02 and $0.15 \mathrm{mg} / 100 \mathrm{ml}$ whereas in patients with skeletal fluorosis the levels varied between 0.02 and $0.19 \mathrm{mg} /$ $100 \mathrm{ml}$. Serum fluoride levels in 500 normal healthy adults ranged between 0.03 to PPM with a mean of $0.08 \mathrm{PPM}$ and they were 0.04 to $0.28 \mathrm{PPM}$ with a mean of 0.16 PPM in 17 fluorotic patients in our laboratory. The urinary levels of fluorides of these 17 fluorotic patients varied between 0.68 to 7.80 PPM with a mean of 3.28 PPM. ${ }^{[18]}$

Bone fluoride content measures the extent of bone fluoride retention and is a useful complement of bone histological studies for the diagnosis of skeletal fluorosis and can be used for the management of fluoride treatment of osteoporosis. In skeletal flurosis fluoride content varies between 6000 and 8400 PPM in bone ash, normal between 500 and $1000 \mathrm{PPM}$ or $\mathrm{mg} / \mathrm{kg}$.

\section{Radionuclide bone scan}

Technetium labeled methylene diphophonate (99mTcMDP) bone scanning measures skeletal metabolism and in skeletal flurosis shows mostly a super scan appearance and in some cases joint abnormalities. Increased tracer activity between the forearm bones 
and diffuse linear tracer activity along the ligamentous attachments may be seen. Concentration of tracer may be noted in the joints such as sacro-iliac, anterior iliac spine where inguinal ligament is attached. ${ }^{[19]}$

\section{Radiology}

In fluorosis radiological findings closely parallel the pathological changes. ${ }^{[1]}$ It is difficult to assert that the density of bone is a result of reactive new bone formation and osteoid as a response to fluoride intoxication. All that can be said is that the density may be quantitative rather than qualitative owing to the increase in the matrix unaccompanied by any increase in mineralization. Bone continues to be formed, but the thickened trabeculae with uncalcified borders are resistant to resorption and so they thicken. In adults, the radiological findings can be categorized into three stages: Stage I - findings mainly confined to the axial skeleton, trabeculae appear rough and bone appears ground glass; Stage II - thick primary trabeculae merge with sclerotic secondary trabeculae to make bone homogenously dense, bone contours become uneven, calcification of paraspinous, sacrospinous and sacrotuberous ligaments; Stage III - axial skeletal bones demonstrate the typical radiological features, calcification is marked at the insertion of muscles and tendons. There can be overlap of radiological features. ${ }^{[19]}$

\section{I maging}

Computer tomography (CT) is the best imaging modality for visualization of bone pathology and provides more details than plain skiagrams. ${ }^{[18]}$ CT visualizes better the direction of the osteophytes, a finding which may help in surgical planning. Spinal canal and root canal stenosis is also better appreciated with CT scan. The calcified ligaments are visualized with much more clarity and earlier than by plain roentgenology, so are the indentations of the epidural space and the alterations in the spinal canal. By reconstruction, CT provides exact dimensions of the ossified intraspinal ligaments such as posterior longitudinal ligament and yellow ligaments. ${ }^{[19]}$

Magnetic resonance imaging (MRI) being noninvasive obviates the difficulties in performing myelography and also delineates soft tissue structures and changes in the spinal cord. It also demonstrates associated abnormalities like psuedomeningocoele. It is worthwhile to image the entire spine, which may demonstrate incidental and interesting pathological lesions peculiar to fluorosis. Fluorotic vertebrae are hypointense in both T1 and T2 weighted images. ${ }^{[19]}$

\section{Differential Diagnosis}

In endemic areas, the diagnosis of dental and skeletal fluorosis does not present much of a problem. In industries, where fluoride toxicity is a known hazard, skeletal fluorosis marked by restriction of spine movements can easily be diagnosed. In early stages of skeletal fluorosis patients complain of arthritic symptoms, which have to be differentiated from other types of arthritis. Diseases that are associated with osteosclerosis also need to be considered in the differential diagnosis. In children and young adults genu valgum deformities have to be distinguished from genu valgum associated with rickets and sometimes with osteodystrophies. When sclerosis of the vertebral column is not marked, calcification of the interosseous membrane of forearm, a radiographic sign of fluorosis, should suggest the diagnosis of fluorosis.

\section{Prevention of Fluorosis}

The best approach to tackle this public health menace will be prevention since no cure is possible once the disease sets in.

Endemic fluorosis: In India over 50\% of ground water sources have excess of fluoride and affect more than 150000 villages. Potable water supply with permissible levels of fluoride though desirable cannot obviously be made available to the vast number of people neither can they be shifted from their area of domicile. Thus there is a rationale to press into service water-purifying or defluoridation plants in those areas. Defluoridation plants are based on Nalgonda method of lime and alum and domestic units were based on filteration of fluoride-rich water with PAC granules, which are activated alumina. Aluminum has been incriminated in the causation of two neurodegenerative diseases Parkinsonism and amyotrophic lateral sclerosis. Defluoridation methods increase aluminum levels of filtered water and hence these methods are not preferred by WHO. Nutrition appears to play a crucial role in the incidence and severity of fluorosis and hence a balanced diet having adequate calcium and vitamins should be promoted to reduce the toxicity of fluoride. ${ }^{[20]}$

Industrial fluorosis: Workers in industries and mining exposed to fluorides should be monitored and it should be ensured that their fluoride content of urine is below 5 PPM. It is observed that skeletal fluorosis would not develop in well-nourished individuals, unless fluoride content of bones exceeds 5000 PPM. 


\section{Medical Treatment}

Patients with skeletal fluorosis, when kept off fluoride intake, register a negative fluoride balance while continuing to excrete large amounts of fluorides for years. It is obvious that excretion of fluorides mobilized from the skeleton through urine and feces is a very slow and prolonged process lasting for many months to even years.

Studies, both in vitro and in vivo, have been done to study the effect of various drugs on the binding of fluoride and also on the excretion of fluoride. In-vitro studies have demonstrated that bone meal, serpentine, dowex, and magnesium compounds could be effective in reducing fluoride content of water. In-vivo experiments in animals showed that calcium, magnesium and aluminum salts could reduce fluoride absorption and also increased its excretion from the body. Serpentine was used to increase fluoride excretion in human fluorosis with some success. ${ }^{[21]}$ Serpentine, a naturally occurring mineral, is chemically a magnesium metasilicate and has enormous capacity to absorb fluoride at a wide range of $\mathrm{pH}$. There is a need to do long-term studies to see the effectiveness of these agents in mobilizing fluoride in human fluorosis. Experiments conducted by Marier found a similarity between magnesium deficiency symptoms and those of fluoride intoxication, which made them suggest that higher intakes of magnesium might prove beneficial in endemic fluorosis cases. ${ }^{[21]}$ Our own clinical and experimental studies confirm their observations and lend support to the view that physiologically magnesium ion has a peculiar affinity for fluoride.

\section{Surgery in Skeletal Fluorosis}

Neurological manifestations of fluorosis are mainly mechanical. However, surgery obviously is of little help in the alleviation of neurological deficits in view of the extensive prevalence of the disease. Surgical decompression is only possible in such of those early cases in which the compression is confined to a limited segment of the vertebral column. But managing even these patients bristles with problems: (1) difficulty in intubation during anesthesia because of the rigid cervical spine; (2) difficulty in positioning of the spine during surgery; (3) high risk for postoperative respiratory complications because of restricted chest movements and reduced vital capacity; laminectomy has to be extensive in view of widespread disease and burr-holes have to be used for removing laminae. However, the results of surgical decompression done in a select group of patients with cervical compression were found to be encouraging but quite discouraging in patients with dorsal compression. The devastating complications seen in patients with dorsal compression may be related to the anatomy of this region and watershed zones of thoracic cord. Lumbar compression rarely necessitates surgical decompression. It is on account of all these reasons that the attitudes towards the use of surgery in the case of spinal compression in skeletal fluorosis vary from cautious optimism to pessimism. ${ }^{[22]}$ However, in recent years with accurate localization of the compression, its extent in both vertical and axial planes by MRI, fiber optic intubation, better illumination and instrumentation during surgery for drilling and removal hard fluorotic bone and postoperative ventilator support made surgery safer and effective in alleviation of mechanical compression in fluorosis.

In recent years surgical approach to certain types of lesions of the cervical spine in fluorosis has changed. Spinal cord compression in the cervical region in skeletal fluorosis may be due to: a) cervical canal stenosis; b) localized calcified ligamentum flavum; c) anterior compression by osteophytes; d) ossification of posterior longitudinal ligament (OPLL); and e) a combination of these lesions. OPLL is very common in skeletal fluorosis and was found in 37 of the 80 people over the age of 40 years in whom cervical spine skiagrams were taken in an endemic village. Posterior approach is indicated in cases of canal stenosis and patients with ossified ligamentum flavum. Major cervical myelopathy precipitated by trivial trauma would also benefit from decompressive laminectomy. OPLL, both long segment and short segment, can be tackled through anterior approach with better results. Earlier, wide posterior laminectomy was the procedure in such cases. Even when posterior approach is undertaken, newer methods such as canal expansive laminaplasty can be an option. This approach obviates the complication associated with the anterior approach that immobilizes the spine and also destabilizing problems associated with extensive laminectomy. Dorsal cord compression in fluorosis is of three types: a) diffuse, extensive where surgery is not beneficial b) localized posterior osteophitic or ossified ligamentous compression, where results are likely to be excellent; and c) localized posterior osteophytes spread over many vertebral levels, though technically feasible for excision, the results are not so rewarding. It is also not known what happens to all those patients after surgery, when they go back to their endemic areas and again start to consume high-fluoride-containing water and food.

\section{References}

1. Reddy DR, Skeletal fluorosis. In: Vinken PJ, Bruyn GW, editors. Handbook of clinical neurology. Vol. 36. Amsterdam: North Holland Publishing Co; 1979. p. 465-504.

2. Susheela AK. Fluorosis: An easily preventable disease through practice of interventions. Delhi: Fluorosis Research and Rural Development Foundation; 2005. 
3. World Health Organization. Environmental Health Criteria 227, Fluorides, Geneva: W.H.O.; 2002. p.14-6.

4. Shortt HE, McRobert GR, Barnard TW, Nayar AS. Endemic fluorosis in the Madras presidency. Indian J Med Res 1937;25:553-68.

5. Pandit CG, Raghavachari TN, Rao DS, Krishnamurthi V. Endemic fluorosis in south India. Indian J Med Res 1940;28:533-58.

6. Naidu MR, Sastry KV, Reddy PK, Reddy DR. Skeletal fluorosis secondary to occult renal disease. Fluoride 1986;19:166-8.

7. Raja Reddy D, Rao CR, Nayak RK, Prasad BC. Estimations of trace elements in drinking water supplies of Podili, Darsi and Kanigiri areas of Andhra Pradesh: Proceedings of the workshop on medical geology, IGCP-454, Nagpur: 2004. p. 384-96.

8. Reddy DR. Some observations of fluoride toxicity. NIMHANS J $1985 ; 3: 79-86$.

9. Koketsu K, Gerard RW. Effects of sodium fluoride on nerve muscle transmission. Am J Physiol 1956;186:278-84.

10. Reddy DR, Deme SR, Sesikaran B, Krishnamurthi D, Rao SH, Ramachandran EP. Muscle and nerve pathology in skeletal fluorosis, Reprinted from: $11^{\text {th }}$ International congress of neurological surgery. Amsterdam The Netherlands: July 6-11, 1997. p. 2125-32.

11. Sesikaran B, Harinarayanan Rao S, Krishnamurthi D, Reddy DR. Studies on skeletal muscle biopsies in endemic skeletal fluorosis. Neurol India 2000;48:187-8.

12. Murthy JM, Anandavalli TE, Reddy DR. Late responses in skeletal fluorosis. Fluoride 1986;19:181-3.

13. Reddy MV, Reddy DR, Ramulu SB, Electromyogrphic studies in fluorosis. Fluoride 1978;11:33-6.

14. Franke J. The spinal cord. In: Symposium on non-skeletal phase of chronic fluorosis. Fluoride 1976;9:30-2.

15. Reddy DR, Murthy JM, Rammohan S. Spinal cord studies in fluorotic dogs. Fluoride 1987;20:290-1.

16. Reddy DR, Rammohan Rao MV, Murthy JM. Urinary fluoride excretion in skeletal fluorosis. Fluoride 1984;17:243-6.

17. Reddy DR, Deme SR. Fluorosis. In: 'Neurology in tropics'. Chopra JS, Sawhney IM, editors. New Delhi: 1998. p. 82-92.

18. Reddy DR, Prasad VS, Reddy J.J. Neuroradiology of skeletal fluorosis. Clin Neurosci Ann Acad Singapore 1993;22:493-500.

19. Liang CK, Cao SR, Epidemiological analysis of endemic fluorosis in China. Environ Carcinogen Ecotoxicol Rev 1997;15:123-38.

20. Reddy DR. Trial of intravenous magnesium hydroxide in fluorosis. Proceedings of the symposium on fluorosis. October 1974. Indian academy of Geoscience, Hyderabad-India. 1977. p. 449-56.

21. Marier JR, The importance of dietary magnesium with particular reference to humans. Fluoride 1969;2:185-7.

22. Reddy DR, Rao BD, Subramanian MV. Results of surgery in spinal compression due to skeletal fluorosis. Indian J Surg 1974;36:30-2.

Accepted on 24-02-2009

Source of Support: Nil, Conflict of Interest: None declared.

\section{Author Institution Mapping (AIM)}

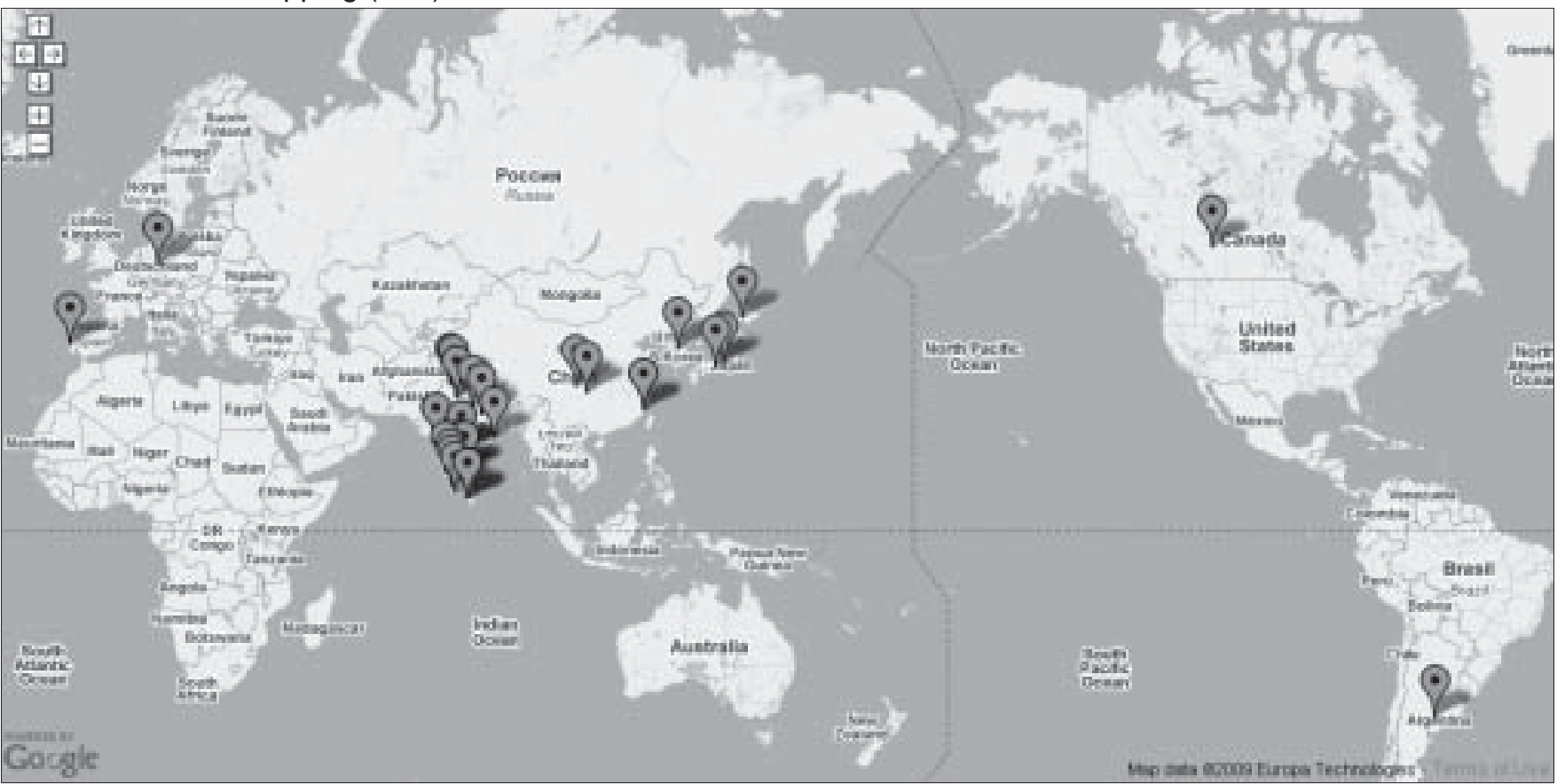

Please note that not all the institutions may get mapped due to non-availability of the requisite information in the Google Map. For AIM of other issues, please check the Archives/Back Issues page on the journal's website. 